\title{
LEFSCHETZ DUALITY \\ AND TOPOLOGICAL TUBULAR NEIGHBOURHOODS
}

BY

\author{
F. E. A. JOHNSON
}

\begin{abstract}
We seek an analogue for topological manifolds of closed tubular neighbourhoods (for smooth imbeddings) and closed regular neighbourhoods (for piecewise linear imbeddings). We succeed when the dimension of the ambient manifold is at least six. The proof uses topological handle theory, the results of Siebenmann's thesis, and a strong version of the Lefschetz Duality Theorem which yields a duality formula for Wall's finiteness obstruction.
\end{abstract}

0. Introduction. Let DIFF, PL, TOP denote the categories of smooth, piecewise linear and topological manifolds and, respectively, smooth, piecewise linear and continuous maps. In each of DIFF and PL, given an imbedding $i: X \rightarrow Y$, there is a natural class $\mathcal{C}(i)$, of closed neighbourhoods of $i(X)$ in $Y$, namely, tubular neighbourhoods in DIFF and regular neighbourhoods in PL. $\mathcal{C}(i)$ has the following properties:

(A) $\mathcal{C}(i)$ is a fundamental system of neighbourhoods of $i(X)$ in $Y$.

(B) If $N \in \mathcal{C}(i), i(X) \hookrightarrow N$ is a simple homotopy equivalence and, if $\operatorname{codim} X$ $\geq 3, \partial N \hookrightarrow N$ induces an isomorphism of fundamental groups.

(C) There is a deformation retraction $r: N \rightarrow i(X)$ such that $\operatorname{Map}(\partial N \stackrel{r}{\rightarrow} i(X))$ $\cong N$ (Map is the mapping cylinder construction).

(D) If $N_{1}, N_{2} \in \mathcal{C}(i)$, there exists $N_{3} \in \mathcal{C}(i)$ such that $N_{3} \subset \operatorname{Int}\left(N_{1} \cap N_{2}\right)$ and an isotopy (in the correct category) $Q: Y \times[0,1] \rightarrow Y$ such that

(i) for each $t \in[0,1], Q_{t}\left|N_{3}=1\right| N_{3}$;

(ii) $Q_{0}=1 Y$ and $Q_{1}\left(N_{1}\right)=N_{2}$.

We seek such a class $\mathcal{C}(i)$ when $i: X \rightarrow Y$ is a locally flat imbedding of a closed manifold $X$ such that $i(X) \cap \partial Y=\varnothing$. We succeed to the following extent.

Theorem 3.6. Let $i: X^{x} \hookrightarrow M^{m}$ be a locally flat imbedding of a closed manifold $X$ into a manifold $M^{m}$ with $x+3 \leq m$ and $m \geq 6$, such that $i(X) \cap \partial M=\varnothing$ ( $X$ and $M$ being assumed only topological manifolds).

Then there is a class $\mathcal{C}(i)$ of closed, locally flat neighbourboods of $i(X)$ in $M$ satisfying properties (A) and (B) above. Moreover, any sucb class then automatically satisfies (D) and the following conditions:

Received by the editors July 30, 1971.

AMS (MOS) subject classifications (1969). Primary 5701, 5705; Secondary 5530, 1820.

Key words and phrases. Class of closed neighbourhoods, derived category, finiteness obstruction, Lefschetz Duality Theorem, collar neighbourhood, topological handle the ory, stable end, Siebenmann thesis. 
(C') $N \in \mathcal{C}(i) \Rightarrow N-i(X) \cong \partial N \times[0,1)$ and

(C') $N \in \mathcal{C}(i) \Longrightarrow i(X)$ is a deformation retract of $N$.

$\left(C^{\prime}\right)$ is a weak form of $(C)$ and, as we shall see, $(C) \Rightarrow\left(C^{\prime}\right) \Rightarrow\left(C^{\prime \prime}\right)$. The question of whether $(C)$ holds in general is interesting.

The method of proof is to use handle theory in the form of [21] on $M-i(X)$, and to forget about $i(X)$, except for requiring that $M-i(X)$ be $1-\mathrm{LC}$ at $i(X)$. If $X$ is a closed manifold and $x+3 \leq m$, this is no more general than requiring $i$ to be a locally flat imbedding [3]. In fact, the method can be applied when $X$ is a compact ANR and $M-i(X)$ is 1-LC at $i(X)$. In general an obstruction arises to completing the method. The obstruction vanishes if and only if $X$ is homotopy equivalent to a finite complex. It is still a classic problem to decide whether or not there exists a compact ANR $X$ not having the homotopy type of a finite complex, with $X$ finite dimensional.

In order to compute the collaring obstruction which arises from the methods of [21] we are forced to prove a stronger form of the Lefschetz Duality Theorem than is usual (2.4). This entails an excursion into the language of derived categories [9].

When $\operatorname{codim} X \leq 1$, Brown [2] proved that a class of neighbourhoods exists with properties (A)-(D) together with the extra condition that the retraction $r: N \rightarrow$ $i(X)$ is a locally trivial bundle with fibre a 1-disc. Kirby [12] announced a proof of the analogous result for $\operatorname{codim} X=2$, but the proof contains a mistake which seems not to have yet been completely corrected [15]. One might ask whether the retraction map $r: N \rightarrow i(X)$ is in general a disc bundle, but this is already false in the PL category $([10],[19])$.

Note that [20] contains a microbundle classification of locally flat imbeddings. However, apart from titles, the intersection of this paper with [20] is just about empty.

The author wishes to express his gratitude to his research advisor Professor C. T. C. Wall who suggested the problem and who has endured several preliminary versions of this paper. The final presentation has gained in clarity after consideration of a communication from C. P. Rourke. The author also wishes to thank the Science Research Council for financial support whilst this work was done, during the period when the author was a research student at the University of Liverpool.

1. Categories of chain complexes and the derived category. For a fuller account of the subject of this section we refer the reader to, for example, [9].

Definition. Let $\mathcal{C}$ be a category and $M$ a class of morphisms in $\mathcal{C}$. A localisation of $\mathcal{C}$ at $M$ is a pair $(\mathscr{D}, \theta)$ where

(i) $\mathfrak{D}$ is a category and $\theta: \mathcal{C} \rightarrow \mathfrak{D}$ is a functor such that, for each $\mu \in M$, $\theta(\mu)$ is an isomorphism.

(ii) For any other such pair $\left(D^{\prime}, \theta^{\prime}\right)$ with the property that $\theta^{\prime}(\mu)$ is an isomorphism, for each $\mu \in M$, there exists a unique functor $\eta: \mathscr{D} \rightarrow \mathfrak{D}^{\prime}$ making the following diagram commute: 


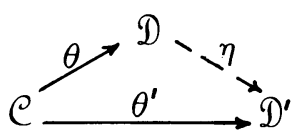

If we do our category theory within a fixed universe then a localisation of $\mathcal{C}$ at $M$ exists for any class of morphisms $M$. (Any two such are clearly isomorphic.) We shall assume this done, so that ontological problems need not concern us. We shall often suppress the localising functor and write $\mathcal{C}\left(M^{-1}\right)$ for the localisation of $\mathcal{C}$ at $M$.

Definition. Let $R$ be a ring (associative with unit). We single out the following categories:

$\mathcal{C}(R)$ : the category of $R$-chain complexes and chain maps.

$\mathcal{C}_{b}(R)$ : the full subcategory of $\mathcal{C}(R)$ consisting of objects $\left(C_{*}, \partial\right)$ such that for some $n \in \mathrm{N}, H_{r}\left(C_{*}\right)=0$ for $|r|>n$ where $H_{*}$ is the homology functor.

$\mathcal{P}(R)$ : the full subcategory of $\mathcal{C}(R)$ consisting of objects $\left(C_{*}, \partial\right)$ with each $C_{r}$ projective such that, for some $n \in \mathbf{N}, C_{r}=0$ for $n<|r|$.

$g(R)$ : the analogue of $\mathscr{P}(R)$ for injective modules.

$\mathfrak{F} \mathcal{P}(R)$ : the full subcategory of $\mathcal{P}(R)$ whose objects $\left(C_{*}, \partial\right)$ have $C_{r}$ finitely generated, for each $r$.

$F(R)$ : the full subcategory of $\mathcal{F P}(R)$ whose objects have (finitely generated) free modules in each dimension.

If $\mathfrak{A} \in\left\{\mathcal{C}(R), \mathcal{C}_{b}(R), \mathcal{P}(R), \mathcal{I}(R), \mathcal{F} \mathcal{P}(R), F(R)\right\}$, denote by $\mathcal{H} \mathfrak{A}$ the homotopy category of $\mathfrak{Q}$, i.e. morphisms are chain homotopy classes of chain maps. Let $H I$ be the class of morphisms in $\mathcal{C}(R)$ which induce isomorphisms of homology groups in all dimensions.

Definition. Define $\operatorname{Der}(R)=\mathcal{C}(R)\left[\mathrm{HI}^{-1}\right]$, and $\operatorname{Der}_{b}(R)=\mathcal{C}_{b}(R)\left[H I^{-1}\right]$. Der $(R)$ is the derived category of $R$. $\operatorname{Der}_{b}(R)$ is, however, more useful as the following theorem shows.

Theorem 1.1. Let \langle\rangle$: \mathcal{C}_{b}(R) \rightarrow \operatorname{Der}_{b}(R)$ be the localisation functor. We have the following equivalence of categories:

$$
\langle\mathcal{P}(R)\rangle \cong\langle\mathscr{G}(R)\rangle \cong \operatorname{Der}_{b}(R) \cong \mathcal{H}(g(R) \cong \mathcal{H} \mathscr{P}(R)
$$

Proof. The statement that $\langle\mathcal{P}(R)\rangle \cong\langle g(R)\rangle \cong \operatorname{Der}_{b}(R)$ is little more than that the category of $R$-modules has enough projectives and injectives (see [9, p. 4045]). The equivalences $\langle\mathscr{P}(R)\rangle \cong \mathcal{H}(\mathcal{P}(R)$ and $\langle\mathscr{g}(R)\rangle \cong \mathcal{H}(\mathscr{g}(R)$ are well known, being the Whitehead Theorem for chain complexes (cf. [24, p. 225]).

Definition. Let $\widetilde{K}_{0}(R)$ be the projective class group of finitely generated projective $R$-modules. Define after Wall ([26], [27]) a function

$$
\sigma: \mathcal{F P}(R) \rightarrow \widetilde{K}_{0}(R)
$$

by 


$$
\sigma\left(C_{*}\right)=\sum_{r \in Z}(-1)^{r}\left\{C_{r}\right\}
$$

where $\{C\}$ is the class of $C$ in $\widetilde{K}_{0}(R)$.

The following is essentially proved in [27, Theorems 5-7]. We just adapt it to our notation.

Theorem 1.2. Let $R$ be a ring and []$: \mathcal{C}_{b}(R) \rightarrow \mathcal{H C}_{b}(R)$ be the obvious func. tor. Then $\sigma$ factors through $\mathcal{H F P}(R)$, thus

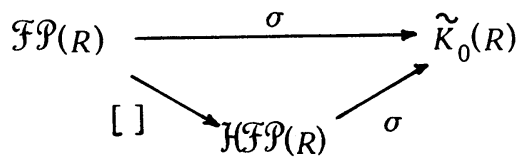

Moreover,

(i) If $C_{*} \in \mathcal{F P}(R)$ then $\left[C_{*}\right] \in \mathcal{H} F(R)$ iff $\sigma\left(C_{*}\right)=0$.

(ii) If $0 \rightarrow X_{*}^{1} \rightarrow X_{*}^{2} \rightarrow X_{*}^{3} \rightarrow 0$ is an exact sequence in $C_{b}(R)$ then any two of $\left[X_{*}^{i}\right], i=1,2,3$, are in $\mathcal{H} \mathfrak{F P}(R)$ if and only if all three are.

(iii) Let $0 \rightarrow X_{*}^{1} \rightarrow X_{*}^{2} \rightarrow X_{*}^{3} \rightarrow 0$ be an exact sequence with all three satis. fying $\left[X_{*}^{i}\right] \in \mathcal{H} \mathcal{F P}(R)$. Then $\sigma\left[X_{*}^{1}\right]+\sigma\left[X_{*}^{3}\right]=\sigma\left[X_{*}^{2}\right]$.

Convention. We agree to confuse $\mathcal{F} \mathscr{P}(R)$ and $F(R)$ with their images in $\operatorname{Der}_{b}(R)$.

2. Chain complex functors and Lefschetz duality. Let $\mathcal{T}$ (resp. $\mathcal{P} \mathcal{S}$ ) denote the category whose objects are second countable, locally compact Hausdorff, locally contractible, finite dimensional topological spaces, whose morphisms are continuous (resp. proper) maps. If $\pi$ is a group, denote by $\mathcal{T}(\pi)$ the subcategory of $\mathcal{T}$ whose objects $X$ are connected, have $\pi_{1}(X)=\pi$, and whose morphisms are maps inducing isomorphisms of $\pi_{1}$. We note that each object in $\mathcal{T}$ is a metrizable ANR and that compact objects in $\mathcal{T}$ exhaust all compact finite dimensional ANR's [1, p. 122]. We shall use the following functors:

$\Delta_{*}: \mathfrak{T} \rightarrow \mathcal{C}_{b}(\mathrm{Z}): \Delta_{*}$ the singular chain complex functor,

$A^{*}: \mathcal{T} \rightarrow \mathcal{C}_{b}(\mathbf{Z}): A^{*}$ the Alexander-Spanier cochain functor [24, Chapter 6],

$\Delta^{*}: \mathcal{J} \rightarrow \mathcal{C}_{b}(\mathbf{Z}): \Delta^{*}()=\operatorname{Hom}_{Z}\left(\Delta_{*}() ; \mathbf{Z}\right)$,

$A_{c}^{*}: \mathfrak{P T} \rightarrow \mathcal{C}_{b}(\mathrm{Z}): A_{c}^{*}$ the subcomplex of $A^{*}$ with compact supports $[24, \mathrm{p}$. 320],

$\Delta_{c}^{*}: \mathfrak{P T} \rightarrow \mathcal{C}_{b}(\mathrm{Z}): \Delta_{c}^{*}$ the subcomplex of $\Delta^{*}$ with compact supports,

$C_{*}: \mathcal{T}(\pi) \rightarrow \mathcal{C}_{b}\left(\mathbf{Z}_{\pi}\right)$ defined by $C_{*}(X)=\Delta_{*}(\tilde{X})$,

$C^{*}: \mathcal{C J}(\pi) \rightarrow \mathcal{C}_{b}\left(\mathrm{Z}_{\pi}\right)$ defined by $C^{*}(X)=A_{c}^{*}(\tilde{X})$, where $\mathcal{C J}(\pi)$ is the full subcategory of $\mathcal{T}(\pi)$ of compact objects. Here $\sim$ denotes 'universal cover' and the $\pi$-action on $C_{*}, C^{*}$ is induced by $\pi$-action on universal covers. The above functors enjoy the following properties [24, Chapter 6]: 
(A) There are natural transformations $r: A^{*}() \rightarrow \Delta^{*}() \tau: A_{c}^{*}() \rightarrow \Delta_{c}^{*}()$ which induce homology isomorphisms on $\mathcal{T}, \mathcal{P} \mathcal{T}$ respectively.

(B) Let $X \in \mathcal{T}$ and suppose $B \subset A \subset X$ are closed subsets. Put

$\Re=\Re(A, B)=\{(U, V)$ is a neighbourhood of $(A, B)$ and $\overline{X-V}$ is compact $\}$. Then the natural map

$$
\lim _{\vec{r}} A^{*}(U, V) \rightarrow A_{c}^{*}(A, B)
$$

induces a homology isomorphism.

We can now make a start on the Lefschetz Duality Theorem.

Definition. Let $X$ be a manifold of dimension $n$. Let $\delta(X) \subset X \times X$ be the diagonal. Let $\sigma$ be the Eilenberg-Zilber equivalence

$$
\sigma: \Delta^{m}() \times() \rightarrow \operatorname{Hom}\left(\Delta_{*}() \times \Delta_{m-*}() ; \mathbf{Z}\right) .
$$

Let $(C, D) \subset X$ and let $i:(X-D, X-C) \times(C, D) \rightarrow(X \times X, X \times X-\delta)$ be the inclusion. For each $U \in \Delta^{n}(X \times X, X \times X-\delta)$ define a chain map

$$
\gamma_{U}: \Delta_{*}(X-D, X-C) \rightarrow \Delta^{n-*}(C, D)
$$

by

$$
\gamma_{U}(x)=\left(\sigma \circ i^{*}(U)\right) / x,
$$

where / is slant product (see [24, p. 287]).

We will also need

Lemma 2.1. Let $X$ be an orientable manifold of dimension n. Let $U \epsilon$ $\Delta^{n}(X \times X, X \times X-\delta(X))$ be an orientation cocycle. Let $(C, D)$ be a compact pair in $X$ with $C \cap \partial X=\varnothing$. Then

$$
\stackrel{\lim }{\rightarrow} \gamma_{U}: \lim _{\rightarrow} \Delta_{*}(X-W, X-V) \rightarrow \underset{\lim }{\rightarrow} \Delta^{n-*}(V, W)
$$

is a chain map inducing bomology isomorphisms, where $\underset{\rightarrow}{\lim }$ is taken for $(V, W) \epsilon$ $r(C, D)$ as defined above.

For a proof see [24, pp. 296-298]. Next we prove

Lemma 2.2. Let $X$ be an orientable manifold of dimension n. Let $U \epsilon$ $\Delta^{n}(X \times X, X \times X-\delta(X))$ be an orientation cocycle. Let $(A, B)$ be a closed pair in $X$ satisfying

(i) $A \cup \partial X, B \cup \partial X, \overline{A-B}, \overline{A-B} \cap B$ are locally contractible,

(ii) $\overline{A-B}$ is compact and $\overline{A-B} \cap X=\varnothing$.

Then $\gamma_{U}: \Delta_{*}(X-(B \cup \partial X), X-(A \cup \partial X)) \rightarrow \Delta^{n-*}(A \cup \partial X, B \cup \partial X)$ is a chain map inducing bomology isomorphisms. 
Proof. Let $\pi^{\prime}$ be a cofinal subsystem of $\pi(\overline{A-B} \cup \partial X,(\overline{A-B} \cap B) \cup \partial X)$ such that each $(V, W) \in \Re^{\prime}$ is an open pair. Put $(C, D)=(\overline{A-B}, \overline{A-B} \cap B)$. We have the following commutative diagram of chain maps in which we want to prove that $\gamma_{U_{1}}$ induces homology isomorphisms.

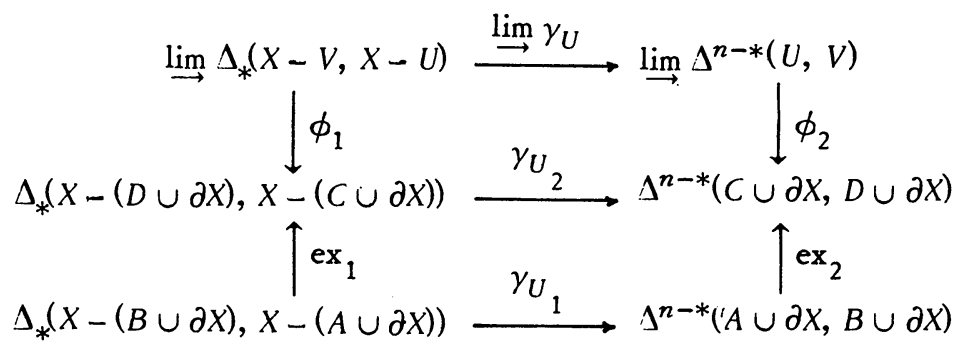

Here ex stands for excision. ex $x_{1}$ is a true excision since $X-(C \cup \partial X)$ is open. $\stackrel{\lim }{\rightarrow} \gamma_{U}$ is an isomorphism on homology by 2.1. $\phi_{1}$ is an isomorphism on homology since $\Delta_{*}$ has compact supports in the terminology of [4], [24]. Thus $\gamma_{U_{1}}$ and $\gamma_{U_{2}}$ induce homology isomorphisms if and only if $\phi_{2}$ and $e_{2}$ do. Accordingly, consider the following commutative diagram of chain maps:

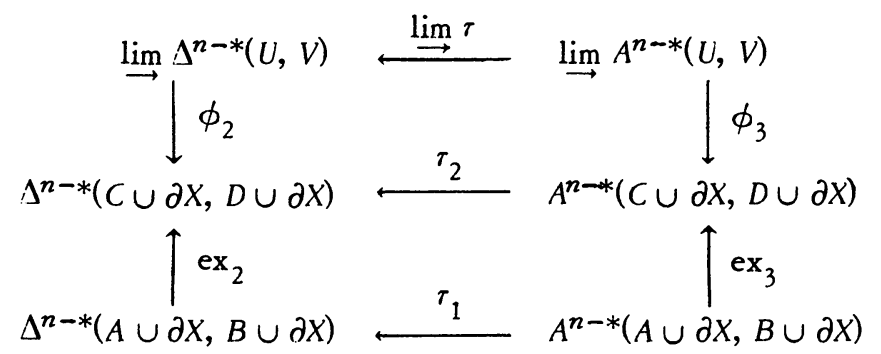

Now $\underset{\lim }{\longrightarrow} \tau, \tau_{2}, \tau_{1}$ induce homology isomorphisms by property (A) above, ex $x_{3}$ is the strong excision for $A^{*}$, thus induces homology isomorphisms as does $\phi_{3}$ by the continuity property for $A^{*}$. Thus $\phi_{2}$ and $e x_{2}$ and hence $\gamma_{U_{2}}$ and $\gamma_{U_{1}}$ induce homology isomorphisms. Q.E.D.

Corollary 2.3. Let $X$ be an orientable manifold of dimension n. Let $U \in$ $\Delta^{n}(X \times X, X \times X-\delta(X))$ be an orientation cocycle. Let $M$ be a closed submanifold of $X$ with $\overline{X-M}$ compact and $X-M \cap \partial X=\varnothing$. Then the chain map $\gamma_{U}$ : $\Delta_{*}(X-M) \rightarrow \Delta^{n-*}(X, M)$ induces bomology isomorphisms.

Proof. Obvious, since the local contractibility condition of 2.2 is satisfied.

Finally we have

Theorem 2.4 (Lefschetz Duality Theorem). Let $X$ be a compact connected manifold with $\partial X$ connected. Suppose $W \subset$ Int $X$ is a closed, connected, locally contractible subset with 


$$
W \longleftrightarrow X \hookleftarrow X-W \longleftrightarrow \partial X
$$

a diagram in $\mathcal{T}(\pi)$. Then $\left\langle C_{*}(X-W)\right\rangle$ and $\left\langle C^{n-*}(X, W \cup \partial X)\right\rangle$ are isomorphic in $\operatorname{Der}_{b}(\mathrm{Z} \pi)$.

Proof. Recall that $C_{*}(K)=\Delta_{*}(\tilde{K})$. Lifting inclusions to universal covers we get

$$
\tilde{W} \hookrightarrow \tilde{X} \hookleftarrow \widetilde{X-\tilde{W}}\lrcorner \tilde{\partial X}
$$

and $\widetilde{X}$ is orientable.

Now put

$$
\begin{array}{r}
\pi=\{M: M \text { is a closed submanifold neighbourhood of } \\
\widetilde{W} \cup \widetilde{\partial X} \text { such that } \widetilde{X}-M \text { has compact closure }\} .
\end{array}
$$

Then $\pi$ acts on $\pi$ via its action on $X$. By 2.3 we get a $Z(\pi)$ chain map which induces homology isomorphisms, where $\underset{\lim }{\longrightarrow}$ is taken over $\Re$,

$$
\stackrel{\lim }{\rightarrow} \gamma_{U}: \stackrel{\lim }{\rightarrow} \Delta_{*}(\widetilde{X}-M) \rightarrow \lim _{\rightarrow} \Delta^{n-*}(\widetilde{X}, M)
$$

Also the obvious $\mathrm{Z}(\pi)$-chain map below induces homology isomorphisms by the compact supports property for $\Delta_{*}$,

$$
\lim _{\rightarrow} \Delta_{*}(\tilde{X}-M) \rightarrow C_{*}(X-(W \cup \partial X)) .
$$

By property (A) above, since we are working in $\mathcal{T}$,

$$
\lim _{\longrightarrow} A^{n-*}(\tilde{X}, M) \stackrel{\lim _{\longrightarrow} \tau}{\longrightarrow} \underset{\lim }{\longrightarrow} \Delta^{n-*}(\tilde{X}, M)
$$

induces homology isomorphisms. Finally by property (B) above,

$$
\phi: \lim _{\rightarrow} A^{n-*}(\tilde{X}, M) \rightarrow A_{c}^{n-*}(\tilde{X}, \tilde{W} \cup \tilde{\partial X})
$$

induces homology isomorphisms and

$$
C^{*}(X, W \cup \partial X)=A_{c}^{*}(\widetilde{X}, \widetilde{W} \cup \widetilde{\partial X})
$$

Thus by (I)-(IV) we have that $\left\langle C_{*}(X-(W \cup \partial X))\right\rangle \cong\left\langle C^{n-*}(X, W \cup \partial X)\right\rangle$ in $\operatorname{Der}_{b}\left(Z_{\pi}\right)$. Taking a collar on $X$ we have $\left\langle C_{*}(X-W)\right\rangle=\left\langle C^{n-*}(X, W \cup \partial X)\right\rangle$. Q.E.D.

Now recall Wall's theory of finiteness conditions for homotopy type $([26],[27])$. We summarize Wall's main theorem thus.

Theorem 2.5. Suppose $X \in \mathcal{T}(\pi)$. Then

(a) $X$ is dominated in bomotopy by a finite complex if and only if $\left\langle C_{*}(X)\right\rangle \epsilon$ $\mathfrak{F} \mathscr{P}\left(\mathbf{Z}_{\pi}\right)$. If $X$ is compact then $X$ is dominated by a finite complex.

(b) If $X$ is dominated by a finite complex, then $X$ is equivalent to a finite complex if and only if 
(i) $\left\langle C_{*}(X)\right\rangle \in F\left(\mathbf{Z}_{\pi}\right)$, or equivalently,

(ii) $\sigma\left(\left\langle C_{*}(X)\right\rangle\right)=0 \in \widetilde{K}_{0}\left(\mathbf{Z}_{\pi}\right)$.

It is customary to write $\sigma(X)=\sigma\left(\left\langle C_{*}(X)\right\rangle\right)$. Let ' - ' denote the involution $\widetilde{K}_{0}\left(\mathrm{Z}_{\pi}\right) \rightarrow \widetilde{K}_{0}\left(\mathbf{Z}_{\pi}\right)$ induced by $\operatorname{Hom}_{\mathbf{Z} \pi}\left(-; \mathbf{Z}_{\pi}\right)$. The next proposition is essentially well known.

Proposition 2.6. Let $X \in \mathcal{T}(\pi)$ be compact. Then

$$
\left\langle C^{*}(X)\right\rangle \cong\left\langle\operatorname{Hom}_{\mathrm{Z} \pi}\left(C_{*}(X): \mathbf{Z}(\pi)\right\rangle \quad \text { in } \operatorname{Der}_{b}(\mathrm{Z} \pi) .\right.
$$

Hence $\left\langle C^{*}(X)\right\rangle \in \mathcal{F P}\left(\mathbf{Z}_{\pi}\right)$ and $\sigma\left(\left\langle C^{*}(X)\right\rangle\right)=\overline{\sigma(X)}$.

Proof. Recall that $C^{*}(X)=A_{c}^{*}(\tilde{X})$ and $C_{*}(X)=\Delta_{*}(\tilde{X})$ and $\tau: A_{c}^{*}(X) \rightarrow \Delta_{c}^{*}(X)$ induces isomorphisms for $X \in \mathcal{T}(\pi)$. Define two presheaves $\Gamma, \Omega$ of $\mathbf{Z}_{\pi}$ chain complexes on $X$ as follows, where $p: \tilde{X} \rightarrow X$ is the covering projection:

(i) $\Gamma^{*}(U)=\Delta_{c}^{*}\left(p^{-1} U\right)$,

(ii) $\Omega^{*}(U)=\operatorname{Hom}_{\mathrm{Z} \pi}\left(\Delta_{*}\left(p^{-1} U\right): \mathrm{Z}_{\pi}\right)$.

Define a $\mathbf{Z}_{\pi}$ chain map $\alpha$ by

$$
\alpha_{U}: \Gamma^{*}(U) \rightarrow \Omega^{*}(U)
$$

with

$$
\alpha_{U}(f)(\sigma)=\sum_{g \in \pi} g f\left(g^{-1} \sigma\right) .
$$

We contend that $\alpha_{x}$ induces an isomorphism on homology. It suffices to check this locally since $X$ is locally contractible and compact. Take a covering $\left(U_{\lambda}\right)$ of $X$ such that, for each $\lambda ; p^{-1}\left(U_{\lambda}\right) \cong U_{\lambda} \times \pi$. We get a commutative triangle

$$
\begin{gathered}
\Delta_{c}^{*}\left(U_{\lambda} \times \pi\right) \stackrel{\alpha}{\longrightarrow} \operatorname{Hom}_{\mathrm{Z} \pi}\left(\Delta_{*}\left(U_{\lambda} \times \pi\right): \mathbf{Z}_{\pi}\right) \\
\frown \\
\operatorname{Hom}_{\mathrm{Z}}\left(\Delta_{*}\left(U_{\boldsymbol{\lambda}} \times \pi\right) ; \mathbf{Z}\right)
\end{gathered}
$$

in which the map $a$ can be viewed as a definition of the $\pi$-action on $\Delta_{c}^{*}\left(U_{\lambda} \times \pi\right)$ and is certainly an isomorphism. Q.E.D.

As a consequence of our Lefschetz Theorem and 2.6 we have

Corollary 2.7. Let $W \in \mathcal{T}(\pi)$ be a compact object. Let $W \hookrightarrow X \hookleftarrow X-W \hookleftarrow \partial X$ be a diagram in $\mathcal{T}(\pi)$ with $X$ a compact manifold of dimension $n$. Then

(i) $X-W$ is dominated by a finite complex,

(ii) $\sigma(X-W)=(-1)^{n+1} \overline{\sigma(W)}$ and $W$ is equivalent to a finite complex iff $X-W$ is.

Proof. (i) Look at the exact sequence 


$$
0 \rightarrow C^{*}(X ; W \cup \partial X) \rightarrow C^{*}(X) \rightarrow C^{*}(W) \oplus C^{*}(\partial X) \rightarrow 0 .
$$

Since $\left\langle C^{*}(X)\right\rangle,\left\langle C^{*}(\partial X)\right\rangle$ and $\left\langle C^{*}(W)\right\rangle \in \mathcal{F} \mathcal{P}\left(\mathbf{Z}_{\pi}\right)$ then so does $\left\langle C^{*}(X, W \cup \partial X)\right\rangle$ by 1.2. By 2.4, $\left\langle C^{n-*}(X, W \cup \partial X)\right\rangle=\left\langle C_{*}(X-W)\right\rangle$ so that $X-W$ is dominated by a finite complex since $\left\langle C_{*}(X-W)\right\rangle \in \mathfrak{F} \mathcal{P}\left(\mathbf{Z}_{\pi}\right)$. Also,

$$
\begin{aligned}
\sigma(X-W) & =\sigma\left(\left\langle C^{n-*}(X, W \cup \partial X)\right\rangle=(-1)^{n} \sigma\left(\left\langle C^{*}(X, W \cup \partial X)\right\rangle\right),\right. \\
& =(-1)^{n}\{\overline{\sigma(X)}-\overline{\sigma(\partial X)}-\overline{\sigma(W)}\} .
\end{aligned}
$$

However, by a result of Kirby-Siebenmann [14], $\sigma(Y)=\overline{\sigma(Y)}=0$ if $Y$ is a compact manifold; hence $\sigma(X-W)=(-1)^{n+1} \overline{\sigma(W)}$. The rest is trivial. Q.E.D.

3. The tubular neighbourhood theorem. The following proposition is due to Hu [11].

Proposition 3.1. Let $(X, A)$ be a topological pair with $A$ closed in $X$ and $X$ an $A N R$. Then $A \hookrightarrow X$ is a cofibration $\Leftrightarrow A$ is an $A N R$.

We shall use the following standard result more than once.

Proposition 3.2. Let $i_{A}: A \hookrightarrow X$ be a cofibration. Then there exists a neighbourbood $U$ of $A$ in $A$, a retraction $r: U \rightarrow A$ and a bomotopy $\left(b_{t}\right): U \rightarrow X$ sucb that

(i) $b_{0}=i_{U}: U \hookrightarrow X$,

(ii) $\left.b_{t}\right|_{A}=i_{A}$ for all $z \in[0,1]$, and

(iii) $b_{1}=i_{A} \circ r$.

Proposition 3.3. Let $X \hookrightarrow N$ be an inclusion of a compact, connected ANR $X$ in a compact connected manifold $N$ such that

(i) $\partial N \cap X=\varnothing$, and

(ii) $N-X=\partial N \times[0,1)$.

Then $X$ is a deformation retract of $N$.

Proof. Let $U$ be a neighbourhood of $X$ in $N, r: U \rightarrow X$ a retraction and $\left(b_{t}\right): U \rightarrow N$ a homotopy satisfying the conclusion of Proposition 3.2. Choose, by hypothesis, a neighbourhood $N^{\prime}$ of $X$ in $N$ such that $X \subset N^{\prime} \subset U$ and such that $\overline{N-N}^{\prime}=\partial N \times[0,1]$. Clearly, $N^{\prime}$ is a deformation retract of $N$. Let $\left(g_{t}\right)_{0 \leq t \leq 1}$ : $N \rightarrow N^{\prime}$ be a deformation retraction from $N$ to $N^{\prime}$. Define $\left(H_{t}\right)_{0 \leq t \leq 1}: N \rightarrow \bar{N}^{\prime}$ by

$$
\begin{array}{ll}
H_{t}=g_{2 t} \text { for } 0 \leq t \leq 1 / 2, \\
H_{t}=b_{2 t-1} \mid N^{\prime} & \text { for } 1 / 2 \leq t \leq 1 .
\end{array}
$$

Then $\left(H_{t}\right)$ is a deformation retraction from $N$ to $X$. Q.E.D.

In the next section we will prove 
Theorem 3.4. Let $M^{m}$ be an open topological manifold with $m \geq 6$. Let $i: X \rightarrow M$ be an imbedding of a compact connected ANR $X$ sucb that $\operatorname{dim} X+3$ $\leq m$ and $M-X$ is 1-LC at $X$. Then there is a closed, locally flat neigbbourbood $N$ of $X$ such that $N-X \cong \partial N \times[0,1)$ if and only if $X$ bas the bomotopy type of a finite complex.

The proof uses 2.7 in an essential way. From this we infer

Theorem 3.5 (Tubular Neighbourhood Theorem). Let $M^{m}$ be an open topological manifold with $m \geq 6$. Let $X$ be a compact connected ANR and let $b: K \rightarrow X$ be a bomotopy equivalence with $K$ a finite complex. Let $i: X \hookrightarrow M^{n}$ be an imbedding with M-X 1-LC at X. Let Wh $(\pi)$ be the Whitebead group of a group $\pi$. Then

(i) There is a closed neighborbood $N$ of $X$ which deformation retracts onto $X$.

(ii) For each $\tau \in \mathbb{W h}(\pi)\left(\pi=\pi_{1}(X)\right)$ there is a closed neigbbourbood $N(\tau)$ of $X$ in $M$ such that $i \circ b: K \rightarrow N(r)$ is a bomotopy equivalence with Whitebead torsion $\tau$; any two such $N^{0}(\tau), N^{1}(\tau)$ are isotopic in $M^{m}$, keeping a third $N^{2}(\tau)$ fixed.

Proof. (i) is immediate from 3.4 and 3.3. (ii) is a well-known application of $s$-cobordism and classification of $b$-cobordisms [25]. Note that the Whitehead torsion statement is well defined since a compact manifold has a well-defined simple homotopy type [14].

To get $N(r)$, take $N$ such that $N$ deformation retracts onto $X$. By the collar neighbourhood theorem, find $N_{1} \hookrightarrow N$ such that $\overline{N-N}_{1} \cong \partial N \times[0,1]$. Now suppose that $b \circ i: K \rightarrow N_{1}$ has torsion $\sigma \in \mathbb{W h}(\pi)$. Decompose $\overline{N-N}_{1}$ as a composite of $b$-cobordisms; thus, $\overline{N-N}_{1}=L_{1} \cup_{\partial L_{1}^{+}} L_{2}$ with $\partial L_{1}^{-}=\partial N_{1}, \partial L_{1}^{+}=\partial L_{2}^{-}$ and $\partial L_{2}^{+}=\partial N$ such that $\partial L_{1}^{-} \hookrightarrow L_{1}$ has Whitehead torsion $(\tau-\sigma)$, so that $\partial L_{2}^{-} \hookrightarrow$ $L_{2}$ has torsion $(\sigma-\tau)$. Define $N(\tau)=N_{1} \cup_{\partial N_{1}} L_{1}$. Then $i \circ b: K \rightarrow N(\tau)$ has torsion $(\tau-\sigma)+\sigma=\tau$. To get uniqueness up to isotopy, take two such neighbourhoods $N^{0}(\tau), N^{1}(\tau)$. Clearly we can find a third $N^{3}(\tau) \subset \operatorname{Int}\left(N^{0}(\tau) \cap N^{1}(\tau)\right)$, and a fourth $N^{2}(\tau)$, such that $\overline{N^{3}(\tau)-N^{2}(\tau)} \cong \partial N^{3}(\tau) \times[0,1]$. Since $\partial N^{3}(\tau) \hookrightarrow$ $\overline{N^{0}(\tau)-N^{3}(\tau)}, \partial N^{3}(\tau) \hookrightarrow \overline{N^{1}(\tau)-N^{3}(\tau)}$ are $s$-cobordisms, then $\overline{N^{0}(\tau)-N^{3}(\tau)} \cong$ $\partial N(\tau) \times[0,1]$ and $\overline{N^{1}(r)-N^{3}(\tau)}=\partial N^{3}(\tau) \times[0,1]$. It is easy to see that $N^{0}(\tau)$ is isotopic to $N^{3}(\tau)$ keeping $N^{2}(\tau)$ fixed; similarly $N^{1}(\tau)$ is isotopic to $N^{3}(\tau)$ keeping $N^{2}(\tau)$ fixed. Thus $N^{0}(\tau)$ and $N^{1}(\tau)$ are isotopic keeping $N^{2}(\tau)$ fixed. We can extend the isotopy to $M$ by the Topological Isotopy Extension Theorem ([13] or [17]). Q.E.D.

Corollary 3.6. Let $i: X^{x} \rightarrow M^{m}$ be a locally flat imbedding of a closed (topological) manifold into a (topological) manifold $M^{m} w i t h x+3 \leq m$ and $m \geq 6$, sucb that $i(X) \cap \partial M=\varnothing$. 
Then there is a class $\mathcal{C}(i)$ of closed, locally flat neighbourboods of $i(X)$ in $M$ satisfying

(I) $\mathcal{C}(i)$ is a fundamental system of neighbourboods of $i(X)$ in $M^{m}$.

(II) If $N \in \mathcal{C}(i)$, then $i(X) \hookrightarrow N$ is a simple bomotopy equivalence and $\partial N \hookrightarrow$ $N$ induces an isomorphism of fundamental groups.

(III) (a) If $N \in \mathcal{C}(i)$ then $N-i(X)=\partial N \times[0,1)$, and

(b) $i(X)$ is a deformation retract of $N$.

(IV) If $N_{1}, N_{2} \in \mathcal{C}(i)$, there exists $N_{3} \subset$ Int $\left(N_{1} \cap N_{2}\right), N_{3} \in \mathcal{C}(i)$, and an isotopy $Q: M \times[0,1] \rightarrow M$ such that

(i) for each $t \in[0,1],\left.Q_{t}\right|_{N_{3} \cup \partial M}=\left.1\right|_{N_{3} \cup \partial M}$ and

(ii) $Q_{0}=1_{M}, Q_{1}\left(N_{1}\right)=N_{2}$.

Proof. Let $\mathcal{C}(i)$ be the class of neighbourhoods satisfying (II). Then by 3.4 and 3.5, $\mathcal{C}(i)$ is nonempty and satisfies (I).

$\mathcal{C}(i)$ now also satisfies (III)(a), for let $N_{0} \in \mathcal{C}(i)$. By (I) there is a sequence $\left(N_{r}\right)_{r \in \mathbf{N}}$ with $N_{r} \in \mathcal{C}(i), N_{r+1} \hookrightarrow$ Int $N_{r}$ and $\bigcap_{r \in \mathbf{N}} N_{r}=i(X)$. By the $s$-cobordism theorem, for each $r \in \mathbf{N}$ we have $\overline{N_{r}-N_{r+1}}=\partial N_{r} \times[0,1]$. Thus $N_{0}-i(X) \cong$ $\partial N_{0} \times[0, \infty) \cong \partial N_{0} \times[0,1)$. Hence $\mathcal{C}(i)$ also satisfies (III)(b) by $3 \cdot 3$. (IV) follows from the same proof as 3.5, taking care to keep the isotopy fixed on $\partial M$. Notice that 3.5 was stated for $X$ connected. If $X$ is not connected, we can take a closed neighbourhood around each connected component of $X$ and apply the method of 3.5 to each neighbourhood in turn, keeping all isotopies fixed on boundaries. Q.E.D.

Appendix: Low dimensional codimension three imbeddings. There are three nontrivial cases of codimension three imbeddings which we have not treated, namely $S^{1} \hookrightarrow M^{4}, S^{1} \hookrightarrow M^{5}$ and $X^{2} \hookrightarrow M^{5}$, where $M, X$ are manifolds.

(I) The case $S^{1} \hookrightarrow M^{4}$. Because of the possible lack of handle structures [22] on 4-dimensional topological manifolds, this case is mysterious though conceivably quite easy. It would be interesting to know to what extent the results of [7] can be generalised.

(II) The cases $X \hookrightarrow M^{5}$ with $\operatorname{dim} X=1,2$. Because of [14], [16] we can say a good deal here. Let $i: X \hookrightarrow M^{5}$ be a locally flat imbedding ( $\left.\operatorname{dim} X \leq 2\right)$. Choose an open neighbourhood $U$, a retraction $r: U \rightarrow X$ such that $j \simeq i \circ r$ (rel $X$ ) where $j: U \hookrightarrow M$ is the inclusion. Thus the classifying map for the topological tangent bundle for $U$ factors through the classifying map for a smooth manifold. Hence $U$ is smoothable. Relative to this smoothing, $i$ is $\epsilon$-isotopic to a smooth imbedding. Hence $i(X)$ has closed, homotopy equivalent neighbourhoods.

(a) $S^{1} \hookrightarrow M^{5}$ : In this case, two close smoothings of $i$ are smoothly isotopic, and the corresponding tubular neighbourhoods are isotopic.

(b) $X^{2} \hookrightarrow M^{5}$ : Here we do not have the information that close smooth imbeddings are smoothly isotopic, though this is conceivable. The question of uniqueness thus remains open. 
4. Proof of Theorem 3.4. For basic information about ends, we refer the reader to [6] or [21]. We recast the definitions of [21] thus.

Definition. Let $\Pi_{1}$ be the fundamental groupoid functor. Let $\xi$ be an isolated end of a topological space $X$. Say that $\mathcal{E}$ is stable if and only if there is a sequence $\left(U_{r}\right)_{r \in \mathbf{N}}$ of neighbourhoods of $\mathscr{E}$ such that

(i) For each $r, \overline{U_{r+1}} \subset$ Int $U_{r}$.

(ii) $\bigcap_{r \in \mathrm{N}} U_{r}=\varnothing$.

(iii) For each $r, \operatorname{Im} Q_{r+1} \stackrel{Q_{r}}{\rightarrow} \operatorname{Im} Q_{r}$ is an equivalence of categories, where $Q_{r+1}: \Pi_{1}\left(U_{r+1}\right) \rightarrow \Pi_{1}\left(U_{r}\right)$ is the functor induced by $U_{r+1} \hookrightarrow U_{r}$. If $\mathscr{G}$ is stable, we define $\pi_{1}(\tilde{G})$ by choosing $\left(X_{r}\right)_{r \in N}$ with $X_{r} \in U$ and $p_{r+1}[0,1] \rightarrow U_{r}$ a path from $X_{r+1}$ to $X_{r}$. Let

$$
\left(p_{r+1}\right)_{*}: \pi_{1}\left(U_{r+1}, X_{r+1}\right) \rightarrow \pi_{1}\left(U_{r}, X_{r}\right)
$$

be the map $\left(p_{r+1}\right)_{*}(\lambda)=\left[p_{r+1}\right] \lambda \cdot\left[p_{r+1}^{-1}\right]$. Then define

Definition. Let $\mathcal{E}$ be an isolated stable end of $X$. A 0 -neighbourhood $U$ of $\mathcal{E}$ is a neighbourhood such that

(i) $U$ is connected,

(ii) $\partial U$ is compact and connected.

A 1 -neighbourhood $U$ of $\mathcal{E}$ is a 0 -neighbourhood such that $\pi_{1}(\mathcal{E}) \cong \pi_{1}(U) \cong$ $\pi_{1}(\partial U)$ (under the obvious maps).

A $k$-neighbourhood $U$ of $\mathcal{E}$ is a 1 -neighbourhood such that $\partial U \hookrightarrow U$ induces isomorphisms of $\pi_{r}$ for $r \leq k$ (for $k \geq 2$ ).

Definition. Let $\mathcal{E}$ be an isolated stable end of $X$. Say that $\mathcal{E}$ is tame iff

(i) every 0 -neighbourhood of $E$ contains a 1 -neighbourhood in its interior, and every 1 -neighbourhood is dominated by a finite complex,

(ii) $\pi_{1}(\mathcal{G})$ is finitely presented.

The above definition contains some redundancies as follows.

Lemma 4.1. Let $\tilde{G}$ be an isolated stable end of a topological manifold $X^{n}$. Suppose $n \geq 6$ (or $n \geq 5$ if $X$ bas a PL structure). Then

(i) every neighbourbood of $E$ contains a 0-neighbourbood,

(ii) if $\pi_{1}(\mathcal{E})$ is finitely presented, then every 0 -neighbourbood of $\mathscr{E}$ contains a 1-neighbourbood,

(iii) $\mathcal{E}$ is tame if and only if $\pi_{1}(\mathcal{E})$ is finitely presented and some 1-neighbourbood is dominated by a finite complex.

Proof. See [21]. The case where $X$ is topological or PL can be obtained by translating Siebenmann's proof, which is given in terms of Morse functions, into handle theoretic terms. We need $n \geq 6$ (rather than $n \geq 5$ ) in the topological case to ensure the existence of topological handle structures ([14], [22]).

We can now state the results of [21] in the following economy size theorem. 
Theorem 4.2. Let $\mathcal{E}$ be a tame end of a topological manifold $M^{n}$ with $n \geq 6$. Then

(i) every neighbourbood of $\mathcal{E}$ contains an $(n-3)$ neighbourbood,

(ii) if $V, V^{\prime}$ are $(n-3)$ neighbourboods of $\mathcal{E}$, then $\sigma(V)=\sigma\left(V^{\prime}\right)$ (we now write $\sigma(\mathcal{E})=\sigma(V) \in \tilde{K}_{0}\left(\mathbf{Z}_{\pi_{1}} \tilde{E}\right)$,

(iii) $\mathcal{G}$ has an $(n-2)$ neighbourbood $V$ if and only if $\sigma(\mathcal{E})=0$,

(iv) if $V$ is an $(n-2)$ neighbourbood of $\mathcal{E}$, then $V=\partial V \times[0,1)(V$ is called a collar neighbourbood).

Proof. See [21] for smooth case. The proof is again handle theoretic and we can use topological handle theory to get the general result. (iii) is the heart of the matter; (ii) uses Lefschetz duality (though an easier version than 2.4) and the fact that $\sigma(Y)=0$ for $Y$ a compact manifold [14]. (iv) follows from the fact that if $V$ is an $(n-2)$ neighbourhood, then $\partial V \hookrightarrow V$ is a homotopy equivalence, plus the classification and invertibility of $b$-cobordisms. But see [21], [23], [25].

In order to prove 3.4 we shall first prove

Lemma 4.3. Let $M^{n}$ be an open topological manifold with $n \geq 6$. Let $A$ be a compact, connected $A N R$ and let $i: A \hookrightarrow M$ be an imbedding sucb that $M-A$ is 1-LC at $A$ and $\operatorname{dim} A+3 \leq n$. Then

(i) $M-A$ bas one isolated, tame end $\mathcal{E}(A)$, corresponding to the removal of $A$, and $\pi_{1}(A) \cong \pi_{1}(\mathcal{G}(A))$.

(ii) $\sigma(\epsilon(A))=(-1)^{n+1} \overline{\sigma(A)}$ and bence $\mathcal{E}(A)$ bas a collar neigbbourbood if and only if $A$ bas the bomotopy type of a finite cell complex.

Proof. We prove first the following

Sublemma 4.4. Let $M^{n}$ be a compact, connected manifold. Let $A \subset$ Int $M^{n}$ be a closed, connected subset with $\operatorname{dim} A \leq n-2$. Then

(i) $M-A$ bas one end, $\overline{M-A}=M$ and $M-A$ is patb connected,

(ii) if $i: M-A \hookrightarrow M$ is the inclusion, then $i_{*}: \pi_{1}(M-A) \rightarrow \pi_{1}(M)$ is surjective,

(iii) if, in addition, $M-A$ is $1-L C$ at $A$, then $i_{*}: \pi_{1}(M-A) \rightarrow \pi_{1}(M)$ is injective, bence an isomorphism.

Proof of Sublemma 4.4. (i) follows from the following information:

(1) Since $\operatorname{dim} A \leq n-1, A$ contains no interior points.

(2) $A$ is connected.

(3) Since $\operatorname{dim} A \leq n-2$, if $M^{\prime}=M-C$ for some compact $C$ with $C \cap A=\varnothing$ then if $M_{A}$ is the connected component of $M^{\prime}$ containing $A, M_{A}-A$ is path connected. (iii) follows easily from Theorem 2 of [5].

It remains to prove (ii). The following is based on a proof of a theorem of [18]. Let \|\| be a norm on $\mathbf{R}^{n}$ and let $E^{n}=\left\{x \in \mathbf{R}^{n}:\|x\| \leq 1\right\}$. Let $\left(U_{x}, b_{x}\right)$ be a 
cover of $M$ with $b_{x}: U_{x} \rightarrow \mathbf{R}^{n}$ a homeomorphism. Put $V_{x}=b_{x}^{-1}\left(E^{n}\right)$. Choose a finite subset $\left(U_{1}, V_{1}\right),\left(U_{2}, V_{2}\right), \ldots,\left(U_{k}, V_{k}\right)$ such that $A \subset \bigcup_{i=1}^{k}$ Int $V_{i}$. If $f: S^{1} \rightarrow M$ is any continuous map, subdivide $S^{1}$ by 1 -simplices $\left(\sigma_{j}\right)$ such that

$$
(\forall j): \dot{f}\left(\sigma_{j}\right) \cap A \neq \varnothing \Rightarrow(\exists r): f\left(\sigma_{j}\right) \subset \text { Int } V_{r}
$$

Now renumber the 1-simplices whose images intersect $A$ thus $\left\{\alpha_{1}, \ldots, \alpha_{k}\right\}$.

Since $\overline{M-A}=M$ and $M-A$ is path connected, we can alter $f$ be an arbitrarily small homotopy to obtain a map $f^{\prime}: S^{1} \rightarrow M$ such that

$$
\begin{gathered}
(\forall j)\left(f\left(\sigma_{j}\right) \cap A \neq \varnothing\right) \Leftrightarrow f^{\prime}\left(\sigma_{j}\right) \cap A \neq \varnothing, \\
(\forall j) f\left(\alpha_{j}\right) \subset \text { Int } V_{r} \Rightarrow f^{\prime}\left(\alpha_{j}\right) \subset \text { Int } V_{r}, \\
f^{\prime}\left(\partial \alpha_{j}\right) \subset V_{r}-A .
\end{gathered}
$$

Now alter $f^{\prime}$ on each $\alpha_{i}$ by choosing a path $p_{i}: I \rightarrow$ Int $V_{r}-A$ between the (at most two) points of $f^{\prime}\left(\partial \alpha_{i}\right)$. We can do this since Int $V_{r}-A$ is path connected. Define $f_{0}^{\prime \prime}=f^{\prime}$ and, for $r \geq 1$, define

$$
f_{r}^{\prime \prime}\left|s^{1}-\alpha_{r}=f_{r-1}^{\prime \prime}\right| s^{1}-a_{r}, \quad f^{\prime \prime} \mid \alpha_{r}=p_{r} .
$$

Put $f^{\prime \prime}=f_{k}^{\prime \prime}$. Then $f^{\prime \prime}\left(S^{1}\right) \subset M-A$. Moreover, since each inclusion $V_{r} \hookrightarrow U_{r}$ is null homotopic, then $f_{r}^{\prime \prime} \simeq f_{r-1}^{\prime \prime}$ in $M$, and hence $f^{\prime \prime} \simeq f^{\prime} \simeq f$ in $M$. Thus $i_{*}: \pi_{1}(M-A)$ $\rightarrow \pi_{1}(M)$ is surjective. Sublemma 4.4 is proved.

We continue with the proof of 4.3 . We show that $\mathcal{E}(A)$ is stable, and has $\pi_{1}(\mathcal{E}(A))=\pi_{1}(A)$. Since $A$ is a compact metrizable finite dimensional ANR it follows from [8] that $A$ is dominated by a finite complex. Thus $\pi_{1}(A)$ is a retract of a finitely presented group, hence (by [26]) is finitely presented. Thus, showing $\pi_{1}(\mathcal{E} A)=\pi_{1}(A)$ also shows that $\pi_{1}(\mathcal{E}(A))$ is finitely presented.

To show that $\mathscr{E}(A)$ is stable and has $\pi_{1}(\mathcal{E}(A))=\pi_{1}(A)$ proceed as follows:

Let $U$ be a neighbourhood of $A$. Construct by 3.2 a sequence $\left(U_{k}\right)$ of neighbourhoods of $A$ such that

(i) each $U_{k}$ is connected, $U_{k+1} \subset U_{k}$ and $\bigcap_{k \in \mathrm{N}} U_{k}=A$,

(ii) for each $k$, if $i_{k}: A \hookrightarrow U_{k}$ is the inclusion, then there exists $r_{k}: U_{k} \rightarrow A$ such that $r_{k}{ }^{\circ} i_{k}=1_{A}$ and such that, for each $k$, the following diagram commutes:

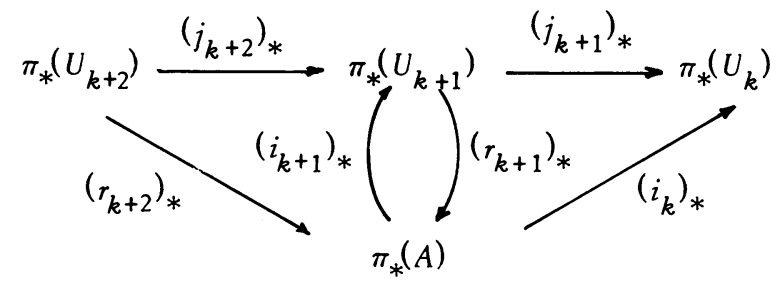

where $j_{k+1}: U_{k+1} \hookrightarrow U_{k}$ is the inclusion.

Now we have 
(a) $\left(i_{k}\right)_{*}$ and $\left(i_{k+1}\right)_{*}$ are injective since they have left inverses $\left(r_{n}\right)_{*}\left(i_{n}\right)_{*}=1_{A}$.

(b) $\operatorname{Im}\left(j_{k+1}\right)_{*}=\operatorname{Im}\left(i_{k}\right)_{*}$ since $\left(j_{k+1}\right)_{*}=\left(i_{k}\right)_{*}\left(r_{k+1}\right)_{*}$ and $\left(r_{k+1}\right)_{*}$ is surjective.

(c) $\left(j_{k+1}\right)_{*}: \operatorname{Im}\left(j_{k+2}\right)_{*} \rightarrow \operatorname{Im}\left(j_{k+1}\right)_{*}$ is an isomorphism, since we have the following commutative triangle in which $\left(i_{k}\right)_{*}$ and $\left(i_{k+1}\right)_{*}$ are isomorphisms (by (a) and (b) above):

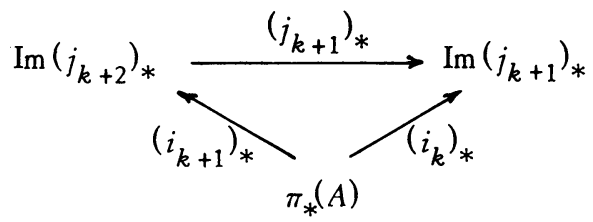

Stability of $\mathcal{E}(A)$ now follows easily, for by 4.4 we have the following commutative diagram for each $k$ :

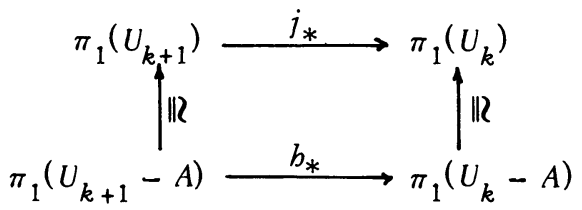

where $b: U_{k+1}-A \hookrightarrow U_{k}-A$ is the inclusion. Hence $\operatorname{Im} b_{*} \cong \operatorname{Im} j_{*} \cong \pi_{1}(A)$ and $\mathcal{E}(A)$ is stable with $\pi_{1}(\mathcal{E}(A)) \cong \pi_{1}(A)$. That $\mathcal{E}$ is tame follows from 2.7 , the existence of 1-neighbourhoods for $\mathcal{E}(A)$ by 4.1 , and the fact that $A$ is dominated by a finite complex. The formula $\sigma(\mathcal{G}(A))=(-1)^{n+1} \overline{\sigma(A)}$ again follows from 2.6. Q.E.D.

Proof of Theorem 3.4. Immediate now by 4.2 and 4.3 .

\section{REFERENCES}

1. K. Borsuk, Theory of retracts, Monografie Mat., Tom 44, PWN, Warsaw, 1967. MR $35 \# 7306$.

2. M. Brown, Locally flat imbeddings of topological manifolds, Ann. of Math. (2) 75 (1962), 331-341. MR 24 \#A3637.

3. J. L. Bryant and C. L. Seebeck III, Locally nice embeddings in codimension 3, Bull. Amer. Soc. 74 (1968), 378-380. MR 36 \#4566.

4. S. Eilenberg and N. E. Steenrod, Foundations of algebraic topology, Princeton Univ. Press, Princeton, N. J., 1952. MR 14, 398.

5. S. Eilenberg and R. L. Wilder, Uniform local connectedness and contractibility, Amer. J. Math. 64 (1942), 613-622. MR 4, 87.

6. D. B. A. Epstein, Ends, Topology of 3-Manifolds and Related Topics (Proc. The Univ. of Georgia Inst., 1961), Prentice-Hall, Englewood Cliffs, N. J., 1962, pp. 110-117. MR $28 \# 1605$.

7. H. Gluck, Embeddings in the trivial range, Ann. of Math. (2) 81 (1965), 195-210. MR 30 \#3456.

8. O. Hanner, Some theorems on absolute neighbourhood retracts, Ark. Mat. 1 (1951), 389-408. MR 13, 266.

9. R. Hartshorne, Residues and duality, Lecture Notes in Math., no. 20, SpringerVerlag, Berlin and New York, 1966. MR $36 \# 5145$.

10. M. W. Hirsch, On tubular neighbourhoods of manifolds. I, II, Proc. Cambridge Philos. Soc. 62 (1966), 177-185. MR 33 \#725; \#726.

11. S. T. Hu, $A$ theorem on the extension of homotopy, Dokl. Akad. Nauk SSSR 57 (1947), 231-234. MR 9, 154. 
12. R. C. Kirby, Codimension-two locally flat embeddings have normal bundles, Topology of Manifolds (Proc. Inst., Univ. of Georgia, Athens, Ga., 1969), Markham, Chicago, Ill., 1970, pp. 416-423. MR $42 \# 8502$.

13. R. C. Kirby and R. Edwards, Deformations of spaces of imbeddings, Ann. of Math. (2) 93 (1971), 63-88.

14. R. C. Kirby and L. C. Siebenmann, On the triangulation of manifolds and the Hauptvermutung, Bull. Amer. Math. Soc. 75 (1969), 742-749. MR $39 \# 3500$.

15. - Codimension two locally flat imbeddings, Notices Amer. Math. Soc. 18 (1971), 983. Abstract $\# 71 \mathrm{~T}-\mathrm{G} 196$.

16. R. K. Lashof and M. Rothenberg, Microbundles and smoothing, Topology 3 (1965), 357-388. MR $31 \# 752$.

17. J. A. Lees, Immersions and surgeries of topological manifolds, Bull. Amer. Math. Soc. 75 (1969), 529-534. MR 39 \#959.

18. D. R. McMillan, A criterion for cellularity in a manifold, Ann. of Math. (2) 79 (1965), 327-337.

19. C. P. Rourke and B. J. Sanderson, An imbedding without a normal microbundle, Invent. Math. 3 (1967), 293-299. MR 36 \#5954.

20. - On topological neighbourhoods, Compositio Math. 22 (1970), 387-424.

21. L. C. Siebenmann, Thesis, Princeton University, Princeton, N. J., 1965.

22. - Disruption of low-dimensional handlebody theory by Rohlin's theorem, Topology of Manifolds (Proc. Inst., Univ. of Georgia, Athens, Ga., 1969), Markham, Chicago, Ill., 1970, pp. 57-76. MR $42 \# 6836$.

23. - On detecting open collars, Trans. Amer. Math. Soc. 142 (1969), 201-227. MR $39 \# 7605$.

24. E. H. Spanier, Algebraic topology, McGraw-Hill, New York, 1966. MR 35 \#1007.

25. J. R. Stallings, On infinite processes leading to differentiability in the complement of a point, Differential and Combinatorial Topology (A Sympos. in Honor of Marston Morse), Princeton Univ. Press, Princeton, N. J., 1965, pp. 245-254. MR 31 \#5213.

26. C. T. C. Wall, Finiteness conditions for CW complexes, Ann. of Math. (2) 81 (1965), 56-69. MR $30 \# 1515$.

27. - Finiteness conditions for CW complexes. II, Proc. Roy. Soc. London Ser. A 295 (1966), 129-139. MR $35 \# 2283$.

DEPARTMENT OF MATHEMATICS, UNIVERSITY COLLEGE LONDON, LONDON W. C. 1, 